\title{
Long-term follow-up of herpetic non-necrotizing retinopathy with occlusive retinal vasculitis and neovascularization
}

\author{
Kim Albert ${ }^{1,2}$, Maureen Masset ${ }^{1,2}$, Sabine Bonnet ${ }^{3}$, François Willermain ${ }^{1,2}$ and Laure Caspers ${ }^{1,2^{*}}$
}

\begin{abstract}
Background: Herpetic necrotizing retinitis is a well-recognized entity. A few cases of herpetic non-necrotizing retinitis were previously reported.

Findings: We retrospectively report two cases of herpetic non-necrotizing retinopathy with a long follow-up. A 19-year-old woman presented with a bilateral diffuse occlusive retinal vasculitis, peripheral neovascularization, and no signs of retinal necrosis. Long-lasting immunosuppressive treatment failed to control the vasculitis until herpes simplex virus type 1 (HSV1) was demonstrated by polymerase chain reaction (PCR) in the aqueous. Acyclovir was then added and immunosuppressive tapered and eventually stopped resulting in a resolution of vasculitis. Only two relapses occurred during the next 6 years and responded rapidly to oral acyclovir.

An 11-year-old boy presented with unilateral scar of stromal keratitis, severe vitritis, and optic disc neovascularization, followed 6 weeks later by peripheral occlusive retinal vasculitis. Therapeutic and diagnostic vitrectomy was performed, and PCR was found to be positive for varicella zoster virus (VZV) in a vitreous specimen. The inflammation responded to oral acyclovir therapy. Recurrence of anterior uveitis with iris depigmentation occurred 4 months after treatment was arrested. After 4 years, he presented again with a recurrence of anterior inflammation and cystoid macular edema (CME). No sign of inflammation was seen for the next 10 years.

Conclusions: These rare cases support the possible role of herpes viruses (HSV or VZV) in occlusive vasculitis without retinal necrosis. PCR was useful to raise the diagnosis and to adapt the treatment. A good response was obtained on oral antiviral therapy.
\end{abstract}

Keywords: Uveitis; Herpes; Virus; Simplex; Zoster; Retinal necrosis; Polymerase chain reaction; Non-necrotizing; Vasculitis

\section{Findings} Introduction

Approximately one third of all uveitis cases are associated with infectious agents [1], and another third remains with an unknown etiology [2]. Polymerase chain reaction (PCR) on intraocular fluids is a reliable and highly sensitive test for the diagnosis of infectious uveitis, including cytomegalovirus (CMV), herpes simplex virus (HSV), and varicella zoster virus (VZV) [3,4].

\footnotetext{
*Correspondence: Icaspers@ulb.ac.be

${ }^{1}$ Department of Ophthalmology, Centre Hospitalier Universitaire Saint-Pierre,

Rue Haute 322, 1000 Brussels, Belgium

${ }^{2}$ Centre Hospitalier Universitaire Brugmann, Place Arthur Van Gehuchten 4, 1020 Brussels, Belgium

Full list of author information is available at the end of the article
}

Several types of herpetic posterior uveitis have been described. The acute retinal necrosis (ARN) syndrome is characterized by peripheral retinal necrosis, retinal arteritis, and a prominent inflammatory reaction in the vitreous and anterior chamber of apparently immunocompetent patients [5]. Progressive outer retinal necrosis syndrome is also a herpetic retinal necrosis with minimal vitreous inflammation occurring in severely immunocompromised patients [6]. A few cases of herpes virus-related nonnecrotizing posterior uveitis have been described. Bodaghi et al. described five patients with posterior uveitis unresponsive to steroids and a positive PCR for herpes viruses (HSV or VZV) in the aqueous. The disease did not respond to conventional therapy with systemic corticosteroids and/or immunomodulatory therapy, but favorable 
response was achieved when therapy was switched to systemic antiviral medication [7].

We report two new cases of herpetic non-necrotizing retinopathy with long follow-up.

\section{Patients and methods}

Patient files were retrieved and retrospectively reviewed. Ophthalmic and systemic data were collected in accordance with the ethic committee of the hospital.

\section{Case 1}

A 19-year-old female was referred in September 1993 for the management of an intravitreal hemorrhage in the right eye, retinal neovascularization of the left eye, and bilateral retinal vasculitis.

Visual acuity (VA) was 20/20 in both eyes. Anterior segment examination was normal. Fundus examination showed severe bilateral occlusive vasculitis (periarteritis and periphlebitis) with intraretinal hemorrhages and peripheral neovascularization. A diffuse vitreal hemorrhage was observed in the right eye while it was more preretinal in the inferior part of left eye. There was no sign of retinal necrosis (Figures 1 and 2).

All known causes of ocular vasculitis as sarcoidosis, presumed tuberculosis-related vasculopathy, systemic lupus erythematosus, antiphospholipid syndrome, Wegener's granulomatosis, Susac's syndrome, multiple sclerosis, Behçet's disease, and syphilis were excluded by an extensive workup. An extensive blood analysis, chest $x$-ray, tuberculin skin test, bronchoalveolar lavage, and gallium scan were performed, but all results returned negative. A diagnosis of bilateral idiopathic occlusive vasculitis was raised. Oral corticosteroid therapy was administered, and laser photocoagulation of the ischemic zones was performed. Therapeutic response remained poor. Therefore, immunosuppressive agents were added. However, despite a strong immunosuppressive treatment, multiple recurrences of occlusive vasculitis occurred with recurrent hemorrhages in the posterior pole, mild vitritis, and rare episodes of anterior chamber inflammation. New ischemic areas were treated with additional laser photocoagulation.
After 3 years follow-up, she developed a cystoid macular edema (CME) in the right eye with VA of 20/25; vision of the left eye remained 20/20. A therapeutic vitrectomy was performed in 1996. Despite this treatment, she continued to develop vasculitis, progressing towards the posterior pole (Figure 3). In 1998, at recurrence of vasculitis associated with anterior cells, an anterior chamber paracentesis was performed, demonstrating HSV 1 by PCR in two aqueous specimens.

Acyclovir therapy was initiated at the dosage $5 \times$ $800 \mathrm{mg}$ daily, tapered, and maintained for 7 months, and immunosuppression was tapered and then arrested. A rapid healing of the vasculitis was obtained (Figure 4). Two relapses of retinal vasculitis were reported about 2 and 4 years after the end of oral acyclovir. Both relapses were treated with oral acyclovir alone (800 mg, 5/day) for 3 weeks, with rapid resolution of the vasculitis and no recurrence until the end of the follow-up in November 2004. Final VA was $20 / 20$ in both eyes.

\section{Case 2}

In 1999, an 11-year-old boy presented with a unilateral inferior occlusive retinal vasculitis, optic disc neovascularization, and severe vitritis, limiting the VA of the right eye to $20 / 50$ at presentation. Slight anterior inflammation with scars of endothelial and deep stromal keratitis and inferior pigmented keratic precipitates (KPs) were also observed in the same eye. Six weeks later, he developed inferior retinal neovascularization and VA dropped to 20/200. Because the patient had a tetralogy of Fallot, the surgeon suspected an endogenous bacterial endophthalmitis, and a diagnostic and therapeutic vitrectomy was performed with section of the fibrovascular membranes and photocoagulation of the ischemic non-necrotized peripheral retina. Evaluation of the vitreous specimens revealed the presence of pigmented macrophages without any bacteria. Moreover, PCR performed on the vitreous specimens returned positive for VZV. Oral treatment with acyclovir $(5 \times 800 \mathrm{mg}$ daily) was administered and VA improved to 20/50. Diffuse leakage of fluorescein was observed from the posterior and peripheral retina as well as

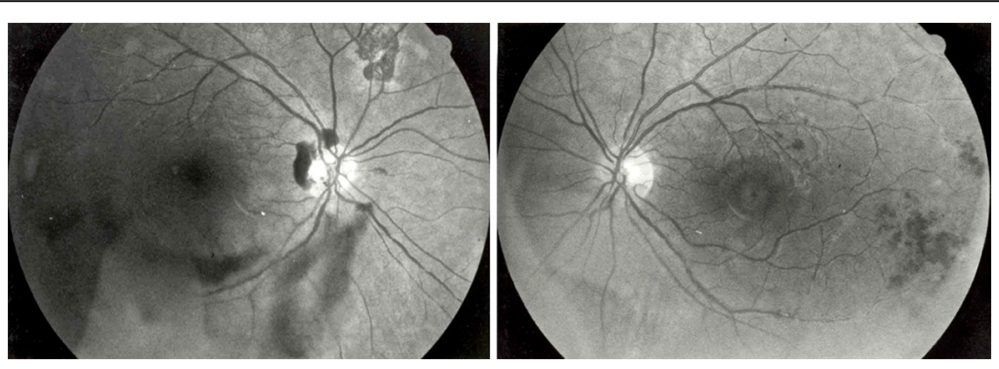

Figure 1 Patient 1: at presentation. Intraretinal hemorrhages and periphlebitis in both eyes. The right eye shows a diffuse intravitreal hemorrhage and a chorioretinal scar nasally. 

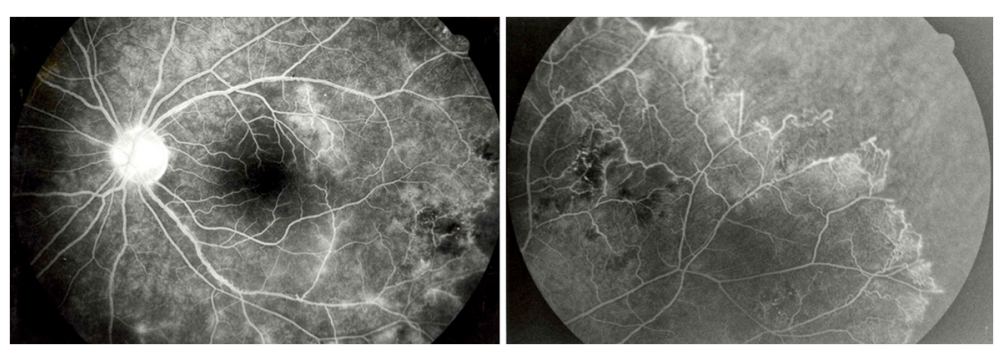

Figure 2 Patient 1: fluorescein angiography at presentation. Diffuse vasculitis and peripheral ischemia of the left eye.

the optic disc (Figure 5). Pigmented cells were still present in the anterior vitreous with $\mathrm{CME}$ and hypertony during the year following vitrectomy, under topical corticosteroids and oral acyclovir. Oral acyclovir (800 mg, 5/day) was administered for 8 months and then replaced by topical acyclovir and topical steroids for another 8 months. A recurrence of anterior uveitis with posterior iris synechiae and iris depigmentation was observed 4 months later. The patient was lost from follow-up and came back in 2003, after 4 years, with an anterior inflammation with granulomatous inferior KPs of the same eye, slight band keratopathy, depigmentation of the inferior iris, posterior iris synechiae, and posterior subcapsular cataract. The left eye remained normal. The patient was treated with oral acyclovir (800 mg, 5/day) during 3 weeks and longlasting non-steroidal anti-inflammatory drops. Slight anterior inflammation and CME remained for one more year. No sign of new active inflammation was seen until 2013. The patient had a final VA of $20 / 200$ (right eye) and 20/20 (left eye).

\section{Discussion}

Case reports of non-necrotizing herpetic uveitis have been reported very rarely $[7,8]$. Bodaghi et al. described five patients with posterior uveitis unresponsive to steroids and whose aqueous aspirates were PCR-positive for HSV or VZV. The clinical presentation consisted of retinal vascular involvement, retinal edema, and papillitis. Anterior inflammation was mild or absent. Posterior segment involvement included features masquerading as Birdshot retinochoroidopathy, Behçet's disease, or idiopathic retinal vasculitis. All patients had chronic inflammation and no necrotizing forms of herpetic retinopathies that progress as an acute disease (ARN or PORN) [7].

Other cases of atypical variants of ARN have been reported, with a slowly progressive ARN and nonnecrotizing forms presenting as vasculitis and/or papillitis or panuveitis [8]. A retinal vasculitis has been described as a complication of primary varicella in an immunocompetent adult [9]. Recently, a case of purely occlusive retinal vasculopathy following varicella zoster infection in an immunocompetent adult, without features of vasculitis or anterior and posterior uveitis, has been published [10]. Chronic herpetic anterior uveitis with focal or multifocal areas of arteriolar sheathing without retinal vascular complications has also been described [11].

We report two new cases of non-necrotizing herpetic retinopathy characterized by occlusive vasculitis with initial neovascularization and a good response to antiviral therapy. The first patient had a history of bilateral recurrent posterior inflammation not responding to steroids and immunosuppressive treatment. This case occurred long before the first case of herpetic nonnecrotizing retinopathy was described, which delayed the diagnosis and antiviral treatment. The second patient presented with a unilateral panuveitis. Both patients had early retinal neovascularization, which has not been described previously [7].

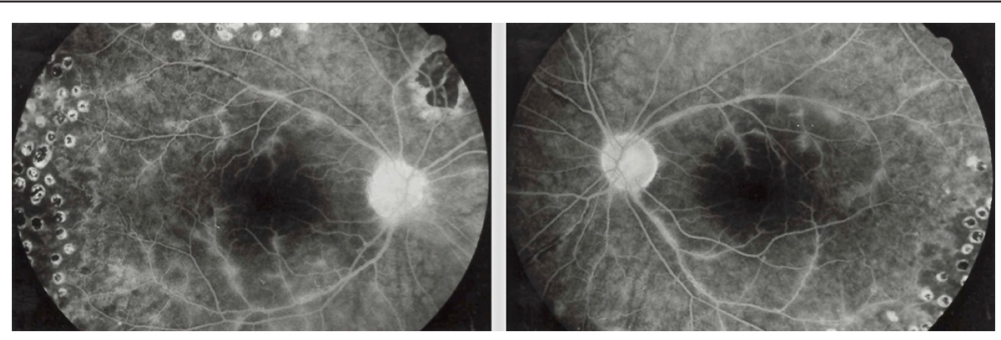

Figure 3 Patient 1: fluorescein angiography during immunosuppressive treatment. Retinal photocoagulation was performed in both eyes. Despite immunosuppressive treatment, vasculitis remained active in both eyes. 

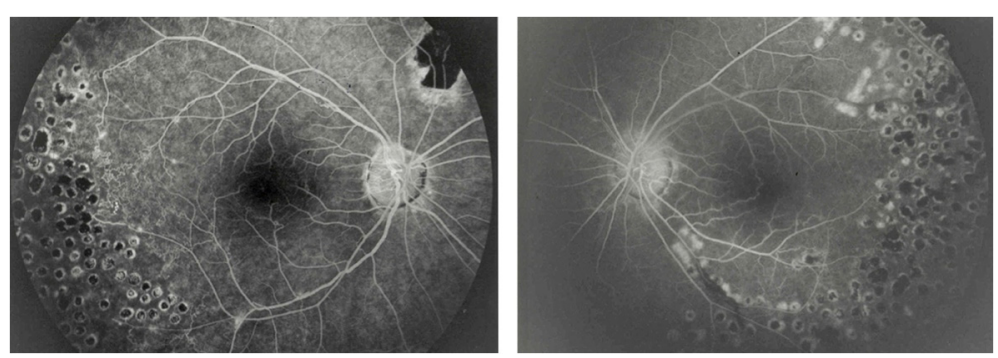

Figure 4 Patient 1: fluorescein angiography after antiviral treatment. No signs of vasculitis in both eyes after antiviral treatment.

A herpetic etiology could be confirmed by PCR analysis of ocular fluids: case 1 had a positive PCR for herpes simplex virus type 1 (HSV1) on the aqueous while case 2 had a positive PCR for VZV on the vitreous. Aqueous analysis was contributory in $86.4 \%$ of patients with necrotizing viral retinopathies [4]. Bodaghi et al. found that DNA amplification from aqueous could detect VZV and HSV in non-necrotizing herpetic retinopathies [7].

A remission was obtained in both cases after reaching the proper etiological diagnosis and initiating antiviral treatment. However, several recurrences occurred after arrest of antiviral treatment. The first patient showed relapses of vasculitis after 2 and 4 years, with fast improvement on oral acyclovir. The second patient showed two recurrences of ipsilateral anterior uveitis. Recurrent anterior uveitis following healed ARN has been reported [12], but to our knowledge, this is the first case of anterior uveitis recurrence after non-necrotizing retinopathy described in the literature. We had previously another patient with a recurrence of HSV2-related acute anterior uveitis demonstrated by PCR, several years after a HSV2related ARN also demonstrated by PCR (L Caspers, unpublished data). Other types of uveitis tend to recur after an ARN episode [12,13]. Even multiple occurrences of ARN separated over long periods can rarely happen in the same eye [14].

Long-standing preventative antiviral therapy could be considered for such patients who present with recurrences of intraocular inflammation.

Our two cases confirm that herpes virus may cause non-necrotizing retinopathy with occlusive vasculitis with early retinal neovascularization. These cases point out the importance of ruling out all infectious, including viral, causes in vasculitis, even in the absence of retinal necrosis, before initiation of immunosuppressive therapy and/or when patients do not respond to immunosuppressive

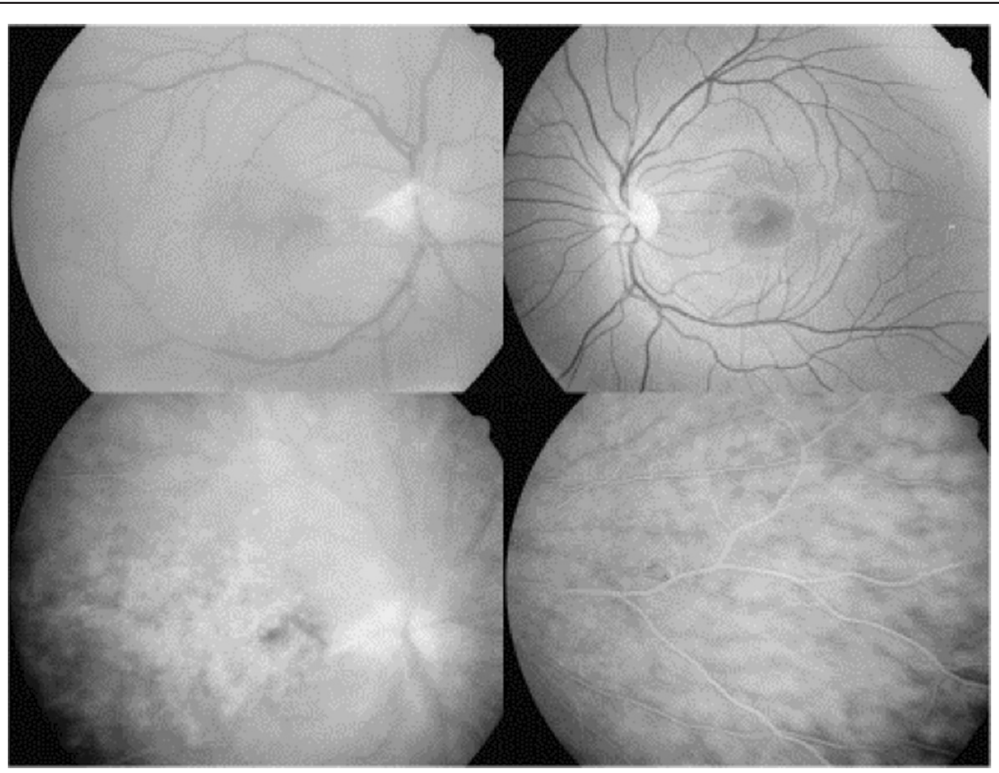

Figure 5 Patient 2. Top left: red free photograph of the right eye with residues of the fibrovascular membrane from the optic nerve partially removed during vitrectomy; top right: red free photograph of the unaffected left eye; down: fluorescein angiography of the right eye showing leakage of dye from the optic disc and the posterior and peripheral inflamed retina. 
treatment. These two cases are, by our knowledge, the first cases of long-term follow-up of non-necrotizing retinopathy.

\section{Consent}

Written informed consent was obtained from the patients for the publication of this report and any accompanying images.

\section{Abbreviations}

ARN: acute retinal necrosis; CME: cystoid macular edema;

CMV: cytomegalovirus; HSV: herpes simplex virus; KPs: keratic precipitates;

PCR: polymerase chain reaction; VA: visual acuity; VZV: varicella zoster virus.

\section{Competing interests}

The authors declare that they have no competing interests.

\section{Authors' contributions}

KA and MM performed the literature review and drafted the manuscript. $\mathrm{SB}$ and $\mathrm{LC}$ managed the case and LC, FW, and SB revised the manuscript critically. All authors read and approved the final manuscript.

\section{Acknowledgements}

The authors have no support from a funding body.

\section{Author details}

'Department of Ophthalmology, Centre Hospitalier Universitaire Saint-Pierre, Rue Haute 322, 1000 Brussels, Belgium. ${ }^{2}$ Centre Hospitalier Universitaire Brugmann, Place Arthur Van Gehuchten 4, 1020 Brussels, Belgium.

${ }^{3}$ Department of Ophthalmology, Centre Hospitalier Regional de la Citadelle, Boulevard du 12ème de Ligne 1, 4000 Liège, Belgium.

Received: 30 June 2014 Accepted: 7 February 2015

Published online: 28 February 2015

\section{References}

1. Bodaghi B, Cassoux N, Wechsler B, Hannouche D, Fardeau C, Papo T et al (2001) Chronic severe uveitis: etiology and visual outcome in 927 patients from a single center. Medicine 80:263-270

2. Bodaghi B (2004) Viral uveitis. J Fr Ophtalmol 27:528-537

3. Scheepers MA, Lecuona KA, Rogers G, Bunce C, Corcoran C, Michaelides M (2013) The value of routine polymerase chain reaction analysis of intraocular fluid specimens in the diagnosis of infectious posterior uveitis. The scientific world journal. doi:10.1155/2013/545149

4. Tran TH, Rozenberg F, Cassoux N, Rao NA, LeHoang P, Bodaghi B (2003) Polymerase chain reaction analysis of aqueous humour samples in necrotising retinitis. $\mathrm{Br} J$ Ophthalmol 87:79-83

5. Holland GN (1994) Standard diagnostic criteria for the acute retinal necrosis syndrome. Executive Committee of the American Uveitis Society. Am J Ophthalmol 117:663-667

6. Forster DJ, Dugel PU, Frangieh GT, Liggett PE, Rao NA (1990) Rapidly progressive outer retinal necrosis in the acquired immunodeficiency syndrome. Am J Ophthalmol 110:341-348

7. Bodaghi B, Rozenberg F, Cassoux N, Fardeau C, LeHoang P (2003) Nonnecrotizing herpetic retinopathies masquerading as severe posterior uveitis. Ophthalmology 110:1737-1743

8. Wensing B, de Groot-Mijnes JD, Rothova A (2011) Necrotizing and nonnecrotizing variants of herpetic uveitis with posterior segment involvement. Arch Ophthalmol 129:403-408

9. Kuo YH, Yip Y, Chen SN (2001) Retinal vasculitis associated with chickenpox. Am J Ophthalmol 132:584-585

10. Narayanan S, Gopalakrishnan M, Giridhar A, Anthony E (2014) Varicella zoster-related occlusive retinal vasculopathy-a rare presentation. Ocul Immunol Inflamm 1:1-4

11. Wickremasinghe SS, Stawell R, Lim L, Pakrou N, Zamir E (2009) Non-necrotizing herpetic vasculitis. Ophthalmology 116:361. doi:10.1016/j.ophtha.2008.09.043

12. Comer M, Young S, Lightman S (2002) Anterior uveitis after healed acute retinal necrosis. Arch Ophthalmol 120(1):88-90
13. Attia S, Khochtali S, Mbarek S, Amor SB, Zaouali S, Messaoud R et al (2013) Acute retinal necrosis associated with central retinal artery occlusion followed after its resolution by keratouveitis. Ocul Immunol Inflamm 21(5):405-407

14. Duker JS, Blumenkranz MS (1991) Diagnosis and management of the acute retinal necrosis (ARN) syndrome. Surv Ophthalmol 35:327-343

\section{Submit your manuscript to a SpringerOpen ${ }^{\circ}$ journal and benefit from:}

- Convenient online submission

- Rigorous peer review

- Immediate publication on acceptance

- Open access: articles freely available online

- High visibility within the field

- Retaining the copyright to your article

Submit your next manuscript at $>$ springeropen.com 